\title{
Correlations among size-related traits are affected by chromosome inversions in an adaptive polymorphism in Drosophila buzzatii
}

\author{
FABIAN M. NORRY*, JUAN C. VILARDI \& ESTEBAN HASSON \\ Departamento de Ciencias Biológicas, Facultad de Ciencias Exactas y Nat., Universidad de Buenos Aires, \\ 1428 Buenos Aires, Argentina
}

\begin{abstract}
Genetic variation in correlations among size-related traits of head, thorax and wings was examined in Drosophila buzzatii, by comparing the correlation pattern of the phenotypic correlation matrix (CP- $\mathbf{R}_{\mathrm{p}}$ ) between inversion karyotypes of the second chromosome. CP- $\mathbf{R}_{\mathrm{p}}$ differed between some karyotypes in a natural population. CP- $\mathbf{R}_{\mathbf{p}}$ in homokaryotypic classes of wild-reared flies, but not in heterokaryotypes, differed from the whole population represented by laboratory-reared flies. Similarity in CP- $\mathbf{R}_{\mathbf{p}}$ was highly significant for a same homokaryotype in two populations. In one of them, the chromosome is polymorphic for four inversions. In the other population, one of the inversions is almost fixed. CP- $\mathbf{R}_{\mathbf{p}}$ was significantly similar between these populations, illustrating that similarity of $\mathbf{C P}-\mathbf{R}_{\mathrm{p}}$ may even occur between populations which have greatly diverged in frequencies of some genotypes affecting correlation patterns. It is suggested that chromosomal inversions are factors affecting genetic correlations among traits known to be phenotypically correlated with fitness components.
\end{abstract}

Keywords: chromosome inversions, Drosophila, phenotypic correlations, size-related traits.

\section{Introduction}

Positive phenotypic correlations of body size with adult fitness components have recently been found in wild populations of some Drosophila species, including the cactophilic fly D. buzzatii. In this species, such a correlation has been detected for three fitness components: mating success (Santos et al., 1988, 1992; Leibowitz et al., 1995; Norry et al., 1995a), fecundity (Santos et al., 1992) and longevity (Santos et al., 1992; Hasson et al., 1993). Recent studies have also shown evidence for the presence of heritable variation in size-related morphometric traits in several wild populations of the same species (Prout \& Barker, 1989; Ruiz et al., 1991; Thomas \& Barker, 1993; Leibowitz et al., 1995). Genetic responses to size-related selection would therefore be expected in $D$. buzzatii populations, if there is a genetic correlation between such heritable variation and the true target(s) of size-related selection.

*Correspondence. E-mail: fmnorry@cbiol.uba.ar
Evolution towards larger adult body size does not appear, however, to be in progress, suggesting that genetic correlations between traits could be a possible factor affecting the response to current selection on size (Leibowitz et al., 1995); for example, there is direct evidence for a negative genetic correlation between adult size and pre-adult survival in D. melanogaster (Partridge \& Fowler, 1993).

Given that genetic correlations yield correlated responses to selection (Falconer, 1989; ch. 19), it is of interest to detect factors affecting genetic correlations among traits phenotypically correlated with fitness components. Chromosomal inversions could be one of such factors in $D$. buzzatii, because the second chromosome inversions affect body size and morphometric traits correlated with it (Ruiz et al., 1991; Hasson et al., 1992; Norry et al., 1995b). This hypothesis is examined in the present study. Specifically, we examine genetic variation in correlations among traits of head, thorax and wings, by comparing the phenotypic correlation matrix $\left(\mathbf{R}_{\mathbf{p}}\right)$ between 
inversion karyotypes within and between populations. If karyotypes (genotypes) differ in the correlation pattern of $\mathbf{R}_{\mathbf{p}}\left(\mathrm{CP}-\mathbf{R}_{\mathrm{p}}\right)$ because of genetic causes in wild flies, this would indicate that inversions are factors affecting genetic correlations through their effects on size-related traits.

\section{Materials and methods}

\section{The data}

To remove any effects of longevity in wild adults, karyotypic variation in $\mathbf{R}_{\mathbf{p}}$ was examined in newly emerged wild flies (NEWFs). They were collected in April 1990 from a population breeding on Opuntia vulgaris at Arroyo Escobar $\left(34^{\circ} 4^{\prime} \mathrm{S}, 58^{\circ} 7^{\prime} \mathrm{W}\right)$, Province of Buenos Aires, Argentina. This population is polymorphic for four inversions on the second chromosome, namely standard (st), j, jz $\mathrm{z}^{3}$ and $\mathrm{jq}^{7}$ (Hasson et al., 1991). Detailed information on the data gathering methods can been found in Norry et al. (1995b), and a brief description follows.

Flies emerging from rotting cladodes of $O$. vulgaris were collected daily, sexed and crossed individually with flies of a homokaryotypic stock. The cytological analysis of eight larvae of the progeny from each cross allowed inference of the karyotype of the wild parent. In total, 354 NEWFs were karyotyped and scored for five traits: thorax length (TL), wing length (WL), wing width (WW), head width (HW) and face width (FW) (see Norry et al., 1995a for details about measurements). An exploratory analysis (see Results) indicated that sample sizes $(N)$ larger than 25 NEWFs permit a relatively precise estimation of $\mathbf{C P}-\mathbf{R}_{\mathbf{p}}$ for these traits. Then, only karyotypic classes with $N>25$ NEWFs were examined. After imposing this constraint the data set contained 30 flies for karyotype $j / s t, 141$ for karyotype $j / j, 136$ for karyotype $\mathrm{j} / \mathrm{jz}^{3}$ and 29 for karyotype $\mathrm{jz}^{3} / \mathrm{jz}^{3}$.

The same traits were also scored in laboratoryreared flies (LRFs), derived from: (i) the Arroyo Escobar population; (ii) the Quilmes population $\left(26^{\circ} 6^{\prime} \mathrm{S}, 65^{\circ} 9^{\prime} \mathrm{W}\right)$, where inversion $\mathrm{j}$ is almost fixed (inversion frequency $=0.98$, Hasson et al., 1995). These flies were the offspring of a random mating performed with the laboratory $F_{2}$ generation of 70 (Arroyo Escobar) or 48 (Quilmes) isofemale lines from wild-inseminated females collected over banana bait buckets. The random mating was performed by releasing three flies of each sex and isofemale line (420 and 288 flies, respectively, for each population) into an egg-collecting chamber $(100 \times 200 \times 300 \mathrm{~mm})$. Samples of 30-40 first-instar larvae were collected daily from this chamber and transferred to $95 \times 20 \mathrm{~mm}$ shell vials with $5 \mathrm{~mL}$ of David's (1962) medium for optimal growth at $25 \pm 1^{\circ} \mathrm{C}$. For each population, 100 randomly chosen flies emerging from these cultures were measured.

\section{Statistical analysis}

All measurements (in ocular units) were $\log _{\mathrm{e}}$-transformed and corrected to remove sexual dimorphism in each trait. This correction was performed by adding to male measurements the difference between male and female means, allowing the pooling of data of both sexes (Schluter \& Smith, 1986). Prior to this, no significant difference in correlations was found between sexes. $\mathbf{R}_{\mathbf{p}}$ was estimated using the Pearson product-moment correlation between traits. Significance levels were adjusted using the sequential Bonferroni test (Rice, 1989).

Severe metric collinearity was apparent in NEWFs, so, therefore, multivariate-normal tests for analyses of covariance matrices do not apply. This study is concerned, not with particular correlations, but with the pattern of $\mathbf{R}_{\mathbf{p}}\left(\mathrm{CP}-\mathbf{R}_{\mathbf{p}}\right)$. Therefore, a randomization procedure free from distributional assumptions, Mantel's test, was applied to compare $\mathbf{C P}-\mathbf{R}_{\mathrm{p}} \mathrm{s}$ by testing the null hypothesis of no matrix correlation. The test statistic is the Pearson correlation coefficient $(r)$ calculated by treating the off-diagonal elements of two matrices as paired observations. In all cases, $1500 \mathbf{R}_{\mathbf{p}}$ permutations were run, using program MXCOMP of the NTSYS package (Rohlf, 1988). The $P$-value reported is the proportion of random permutations with a $r$-value as high as, or higher than, that observed.

\section{Results}

Phenotypic correlations in NEWFs are given in Table 1 for each karyotype examined. All correlations are positive (Table 1). Although phenotypic correlations were typically higher in wild flies than in LRFs (Table 1), Mantel's test did reveal significant similarity of $\mathbf{R}_{\mathbf{p}}$ between NEWFs of Arroyo Escobar (the total sample of 354 NEWFs) and LRFs derived from both populations, Arroyo Escobar $(r=0.60$, $P<0.05)$ and Quilmes $(r=0.80, P<0.01)$.

In NEWFs of Arroyo Escobar, significant or marginally significant similarity of CP- $\mathbf{R}_{p}$ was verified among some karyotypes: $\mathrm{j} / \mathrm{j}, \mathrm{j} / \mathrm{jz} \mathrm{z}^{3}$ and $\mathrm{j} / \mathrm{st}$ (Table 2). However, no similarity in CP- $\mathbf{R}_{\mathbf{p}}$ was detected between homokaryotypes (Table 2), and karyotype $\mathrm{jz}^{3} / \mathrm{jz}^{3}$ differs from the remaining ones (see below). Heterokaryotypes in this natural population, but not homokaryotypes, show a CP- $\mathbf{R}_{\mathbf{p}}$ similar to 
the whole population represented by LRFs (LRF-AE, Table 2).

Given that $\mathbf{R}_{\mathrm{p}}$ for karyotype $\mathrm{j} / \mathrm{j}$ was estimated using a fairly large sample size (141 NEWFs), very precise estimates were available for the comparisons between this karyotype in NEWFs $(N=141)$ and LRFs $(N=100)$. First, the karyotypic influence on CP- $\mathbf{R}_{\mathbf{p}}$ in Arroyo Escobar was also verified by the result that NEWFs carrying the $\mathrm{j} / \mathrm{j}$ karyotype exhibit no matrix similarity with LRFs representing the whole population of Arroyo Escobar (LRF-AE, Table 2), where CP- $\mathbf{R}_{\mathbf{p}}$ would also be affected by the remaining inversions. Secondly, highly significant similarity was verified only in the comparison between NEWFs carrying the $\mathrm{j} / \mathrm{j}$ karyotype in Arroyo Escobar and LRFs derived from Quilmes (Table 2), as expected by the fact that inversion $\mathrm{j}$ is almost fixed in Quilmes (see Materials and methods).

\section{An analysis of empirical results on the statistical power}

The permutation test allows the $H_{0}$ hypothesis of independence to be tested without relying on formal null distributions by evaluating the probability of obtaining a $r$-value as high or higher than that obtained with the observed data. When $H_{0}$ is rejected $(P<0.05)$, it cannot be ascertained whether the matrices are equal, proportional or merely similar in pattern to one another (Cowley \& Atchley, 1992), so that results in Table 2 may imply karyotypic variation conservatively suggested. However, the test does not consider the precision with which the elements of the matrices are estimated. Hence, Mantel's test will have low statistical power if it is applied to matrices with poorly estimated elements (Cowley \& Atchley, 1992). In order to evaluate whether this bias associated with the sample size $(N)$ is expected to be severe in Table 2, ten independent samples were obtained (from Arroyo Escobar) of 25 NEWFs each. The $\mathbf{R}_{\mathrm{p}}$ matrix for each sample was obtained (data not shown). Each of these $\mathbf{R}_{\mathrm{p}} \mathrm{s}$ was compared with the overall $\mathbf{R}_{\mathbf{p}}$ obtained by pooling the nine other samples (excluding each sample from the pooled one in each of the ten comparisons). Using Mantel's test, the ten $\mathbf{R}_{\mathbf{p}} \mathbf{s}$ estimated for these samples $(N=25)$ were all significantly similar to their respective overall $\mathbf{R}_{\mathbf{p}}$

Table 1 Phenotypic correlations between traits are given for (a) homokaryotypes and (b) heterokaryotypes of the second chromosome in a sample of wild Drosophila buzzatii emerging from Opuntia cladodes taken at Arroyo Escobar. (c) Correlations are also shown for laboratory-reared flies (LRF) representing two whole populations: Arroyo Escobar (LRF-AE) and Quilmes (LRF-Q). Abbreviations of traits are explained in the text. $P$-values were adjusted by the sequential Bonferroni method

\begin{tabular}{|c|c|c|c|c|c|}
\hline & TL & WL & WW & HW & FW \\
\hline \multicolumn{6}{|c|}{ (a) Homokaryotypes: $\mathrm{j} / \mathrm{j}$ (below); $\mathrm{jz} / \mathrm{j} \mathrm{z}^{3}$ (above) } \\
\hline TL & & $0.824^{* * *}$ & $0.898^{* * *}$ & $0.879^{* * *}$ & $0.757^{* * *}$ \\
\hline WL & $0.775^{* * *}$ & & $0.843^{* * *}$ & $0.791^{* * *}$ & $0.844^{* * *}$ \\
\hline WW & $0.801^{* * *}$ & $0.777^{* * *}$ & & $0.873^{* * *}$ & $0.804^{* * *}$ \\
\hline HW & $0.776^{* * *}$ & $0.727^{* * *}$ & $0.712 * * *$ & & $0.762 * * *$ \\
\hline FW & $0.697^{* * *}$ & $0.681^{* * *}$ & $0.651^{* * *}$ & $0.795^{* * *}$ & \\
\hline \multicolumn{6}{|c|}{ (b) Heterokaryotypes: st/j (below); $\mathrm{j} / \mathrm{jz} \mathrm{z}^{3}$ (above) } \\
\hline TL & & $0.845^{* * *}$ & $0.815^{* * *}$ & $0.815^{* * *}$ & $0.918 * * *$ \\
\hline WL & $0.774^{* * *}$ & & $0.784 * * *$ & $0.715^{* * *}$ & $0.749 * * *$ \\
\hline WW & $0.897^{* * *}$ & $0.860^{* * *}$ & & $0.672^{* * *}$ & $0.699 * * *$ \\
\hline HW & $0.860^{* * *}$ & $0.741^{* * *}$ & $0.782^{* * *}$ & & $0.840 * * *$ \\
\hline FW & $0.914^{* * *}$ & $0.749^{* * *}$ & $0.795^{* * *}$ & $0.920^{* * *}$ & \\
\hline \multicolumn{6}{|c|}{ (c) LRF-AE (below); LRF-Q (above) } \\
\hline TL & & $0.449^{*}$ & $0.603^{* *}$ & $0.511^{* *}$ & $0.398^{*}$ \\
\hline WL & $0.506^{* *}$ & & $0.332^{*}$ & 0.258 & 0.249 \\
\hline WW & $0.368^{*}$ & $0.497^{* *}$ & & $0.392^{*}$ & 0.268 \\
\hline HW & $0.405^{*}$ & 0.196 & $0.355^{*}$ & & $0.669^{* * *}$ \\
\hline FW & $0.532 * *$ & $0.347^{*}$ & $0.375^{*}$ & $0.739 * * *$ & \\
\hline
\end{tabular}

${ }^{*} P<0.05 ;{ }^{* *} P<0.01 ;{ }^{* * *} P<0.005$. 
Table 2 Matrix correlations $(r)$ measuring similarity in the pattern of $\mathbf{R}_{\mathbf{p}}$ s for second chromosome karyotypes in newly emerged wild Drosophila buzzatii of Arroyo Escobar, and between these karyotypic classes and laboratory-reared flies (LRF) representing two whole populations: Arroyo Escobar (LRF-AE) and Quilmes (LRF-Q). $P$-values indicate the probability of obtaining a $r$-value as high as or higher than that observed. 1500 matrix permutations were performed for all tests

\begin{tabular}{lcccccc}
\hline & & $\mathrm{st} / \mathrm{j}$ & $\mathrm{j} / \mathrm{j}$ & $\mathrm{j} / \mathrm{jz}^{3}$ & $\mathrm{jz}^{3} / \mathrm{jz} \mathrm{j}^{3}$ & LRF-AE \\
\cline { 3 - 7 } $\mathrm{j} / \mathrm{j}$ & $r$ & 0.50 & & & & \\
$\mathrm{j} / \mathrm{jz}^{3}$ & $P$ & $(0.07)$ & & & & \\
& $r$ & 0.64 & 0.69 & & & \\
$\mathrm{jz} \mathrm{z}^{3} / \mathrm{jz} z^{3}$ & $P$ & $(0.05)$ & $(0.04)$ & & & \\
& $P$ & -0.14 & 0.28 & -0.13 & & \\
LRF-AE & $r$ & 0.69 & 0.41 & 0.67 & -0.43 & \\
& $P$ & $(0.03)$ & $(0.18)$ & $(0.02)$ & $(0.09)$ & \\
LRF-Q & $r$ & 0.72 & 0.78 & 0.68 & 0.11 & 0.62 \\
& $P$ & $(0.03)$ & $(0.005)$ & $(0.03)$ & $(0.44)$ & $(0.03)$ \\
\hline
\end{tabular}

Values in italic indicate significant correlations between $\mathbf{R}_{\mathrm{p}} \mathbf{s}$.

$(N=225): 0.87>r>0.65, P<0.05,1500$ random permutations were performed for all ten tests. This indicates that severe bias in Table 2 is unlikely, because only karyotypic classes with $N>29$ NEWFs were included in the study (see Materials and methods).

The definition of the rejection zone in Mantel's test, on the other hand, suggests that a high and negative matrix correlation $(r)$ will generate a low $P$-value. This is confirmed in Table 2, for example, for the comparison between $\mathrm{jz}^{3} / \mathrm{jz}^{3}$ and LRF-AE. However, the $H_{0}$ hypothesis (the two matrices are not correlated) is tested against the $H_{1}$ hypothesis that the two matrices are positively correlated. This implies that negative $r$-values reported in Table 2 should, in fact, be associated to higher $P$-values than positive $r$-values. It should be noted, however, that $P$-values generated by Mantel's test for negative $r$-values were still 'nonsignificant' at the usual 0.05 level (Table 2), indicating a lack of overall similarity between $\mathbf{R}_{\mathbf{p}}$ s. In fact, what is clear here is that $r$-values may be negative if the examined matrices differ in the correlation pattern.

\section{Discussion}

The phenotypic correlation pattern for a suite of size-related traits of head, thorax and wings, is heterogeneous between inversion karyotypes of the second chromosome in a wild population of D. buzzatii. Because any effects of age were experimentally removed, these results do not confound direct karyotypic effects with indirect effects caused by differential longevity related to body size (see Ruiz et al., 1991; Norry et al., 1995b).

Although morphometric effects of chromosome inversions have been observed in some insects, including Drosophila species (Krimbas, 1967; Prevosti, 1967; Stalker, 1980; Ruiz et al., 1991; Bitner-Mathe et al., 1995), to our knowledge, no study has examined the possible effects on the correlation among traits. Because phenotypic correlations are the weighted sums of genetic and environmental correlations (Falconer, 1989), the finding that CP- $\mathbf{R}_{\mathbf{p}}$ may differ greatly between inversion genotypes (karyotypes) within and between populations (Table 2) strongly suggests that inversions are factors affecting the genetic correlation among traits. In addition, the data also suggest that CP- $\mathbf{R}_{\mathbf{p}}$ was not altered by environmental correlations, which are the correlations attributable to environmental deviations plus nonadditive genetic deviations (Falconer, 1989). First, phenotypic correlations are notably higher in NEWFs than in LRFs (Table 1), but $\mathbf{C P}-\mathbf{R}_{\mathbf{p}}$ was highly significantly similar both between NEWFs and LRFs representing the whole population and between karyotype $\mathrm{j} / \mathrm{j}$ in NEWFs and the same karyotype in LRFs derived from Quilmes. Thus, whereas the results suggest that the natural environment is much more variable than the laboratory one, at least for karyotype $j / j$ (and for the set of measured traits), environmental deviations in nature do not substantially alter CP-R. $\mathbf{R}_{\mathbf{p}}$. This finding does not imply that $\mathbf{R}_{\mathbf{p}}$ is independent of environmental deviations, but supports the hypothesis that homokaryotypic variation in CP- $\mathbf{R}_{\mathrm{p}}$ is not only an environmental variation, but partially genetic. Nonadditive effects of the second chromosome inversions, on the other hand, would be apparently very weak on sizerelated traits in D. buzzatii, as most of the phenotypic variance among karyotypes is explained by a linear regression of trait on chromosome dose (Ruiz et al., 1991; Hasson et al., 1992; Norry et al., 1995b). Secondly, phenotypic correlations among morphometric traits often show correlation patterns similar to their genetic counterparts, indicating that CP- $\mathbf{R}_{\mathbf{p}}$ is not typically altered by environmental deviations and/or nonadditive genetic deviations (Cheverud, 1988; Roff, 1995, 1996; Simons \& Roff, 1996).

In $D$. buzzatii, at least a fraction of the heritable variation in size-related traits would be determined by the additive contribution of the second chromosome inversions to the phenotypic variance in body 
size (Ruiz et al., 1991; Hasson et al., 1992; Norry et al., 1995b). However, although size-related selection is substantial in wild D. buzzatii populations, the selection response depends, not only on the heritabilities of traits considered singly, but also on the genetic correlations among all the traits of which the phenotype is composed (Lande, 1979; Falconer, 1989). In the Arroyo Escobar population, the five traits studied here are genetically correlated with one another (Norry et al., 1997), as expected by considering that the inversion polymorphism contributes to their respective additive genetic variances (Norry et al., 1995b). In addition, these traits phenotypically covary with mating success and longevity, and face width would be a direct target of sizerelated sexual selection (Norry et al., 1995a; Norry \& Vilardi, 1996). A correlated response to such selection could then be partially influenced by chromosome inversions if, as suggested here, they also affect the genetic correlation among size-related traits. The importance of such an effect is amplified by the fact that these chromosome arrangements are also differentially correlated with preadult and adult survival in the same population (Hasson et al., 1991; Norry et al., 1995b). This information combined with the present data indicates that inversions also affect genetic correlations among preadult survival and size-related adult traits. Overall, it was previously demonstrated that these chromosome arrangements additively affect adaptive traits and fitness components in wild populations of D. buzzatii (Ruiz et al., 1986, 1991; Hasson et al., 1991; Norry et al., 1995b). Therefore, these chromosome arrangements may influence the genetic correlation among adaptive traits, as suggested in this morphometric study. This is exactly what the adaptive basis of the inversion polymorphism would imply.

Because genetic correlations are sensitive to changes in gene frequencies (Turelli, 1988; Falconer, 1989), variation in genetic correlations and CP- $\mathbf{R}_{\mathbf{p}}$ would be expected between populations such as Arroyo Escobar and Quilmes, which differ greatly in inversion frequencies and have diverged, genetically, in body size and shape (Norry, 1995). Yet, CP- $\mathbf{R}_{\mathbf{p}}$ is similar between these populations (Table 2), illustrating that similarity of $\mathrm{CP}-\mathbf{R}_{\mathrm{p}}$ may even occur between populations which have greatly diverged in frequencies of some genotypes affecting correlation patterns. In order to test hypotheses about the dynamics of genetic covariance patterns (see Turelli, 1988), the next step of this work would be to compare estimates of these genetic parameters between populations which differ in inversion frequencies.

(C) The Genetical Society of Great Britain, Heredity, 79, 585-590.

\section{Acknowledgements}

We thank J. Fanara and C. Rodriguez for their assistance in field and laboratory work. We wish to thank an anonymous reviewer for constructive comments on an early version of the manuscript. F.M.N. is a fellow and J.C.V. and E.H. are members of Consejo Nacional de Investigaciones Científicas y Técnicas (Argentina). This work was supported by University of Buenos Aires grant EX 050 and 099 to E.H.

\section{References}

BARKER, J. S. F. AND KREBS, R. A. 1995. Genetic variation and plasticity of thorax length and wing length in Drosophila aldrichi and D. buzzatii. J. Evol. Biol., 8, 689-709.

BITNER-MATHE, B. C., PEXIOTO, A. A. AND KlaCZKo, L. B. 1995. Morphological variation in a natural population of Drosophila mediopunctata: altitudinal cline, temporal changes and influence of chromosomal inversions. Heredity, 75, 54-61.

CHEVERUD, J. M. 1988. A comparison of genetic and phenotypic correlations. Evolution, 42, 958-968.

COWLEY, D. E. AND ATCHLEY, w. R. 1992. Comparison of quantitative genetic parameters. Evolution, 46, 1965-1967.

DAVID, J. 1962. A new medium for rearing Drosophila in axenic conditions. Dros. Inf. Serv, 36, 128.

FAlCONER, D. S. 1989. Introduction to Quantitative Genetics, 3rd edn. Wiley, New York.

HASSON, E., VILARDI, J. C., NAVEIRA, H., FANARA, J. J., RODRIGUEZ, C., REIG, O. A. AND FONTDEVILA, A. 1991. The evolutionary history of Drosophila buzzatii. XVI. Fitness component analysis in an original natural population from Argentina. J. Evol. Biol., 4, 209-225.

HASSON, E., FANARA, J. J., RODRIGUEZ, C., VILARD1, J. C., RElG, O. A. AND FONTDEVILA, A. 1992. The evolutionary history of Drosophila buzzatil. XXIV. Second chromosome inversions have different average effects on thorax length. Heredity, 68, 557-563.

HASSON, E., FANARA, J. J., RODRigUEZ, C., VILARDl, J. C., REIG, O. A. AND FONTDEvilA, A. 1993. The evolutionary history of Drosophila buzzatii. XXVII. Thorax length is positively correlated with longevity in a natural population from Argentina. Genetica, 92, 61-65.

HASSON, E., RODRIGUEZ, C., FANARA, J. J., NAVEIRA, H., REIG, O. A. AND FONTDEVILA, A. 1995. The evolutionary history of Drosophila buzzatii. XXVI. Macrogeographic patterns of inversion polymorphism in New World populations. J. Evol. Biol., 8, 369-384.

KR1MBAS, C. B. 1967. The genetics of Drosophila subobscura populations. III. Inversion polymorphism and climatic factors. Mol. Gen. Genet., 99, 133-150.

LANDE, R. 1979. Quantitative genetic analysis of multivariate evolution, applied to brain:body size allometry. Evolution, 33, 402-416. 
LEIBOWITZ, A., SANTOS, M. AND FONTDEVILA, A. 1995. Heritability and selection on body size in a natural population of Drosophila buzzatii. Genetics, 141, 181-190.

LOFSVOLD, D. 1986. Quantitative genetics of morphological differentiation in Peromyscus. I. Test of the homogeneity of genetic covariance structure among species and subspecies. Evolution, 40, 559-573.

NORRY, F. M. 1995. Selección Sexual Relacionada con el Tamaño Corporal, Parámetros Genetico-Cuantitativos Multivariados, y Divergencia Morfométrica Multivariada entre Poblaciones de dos Especies Cactófilas del Género Drosophila. Ph.D. Thesis, Universidad de Buenos Aires.

NORRY, F. M. AND VILARD1, J. c. 1996. Size-related sexual selection and yeast diet in Drosophila buzzatii (Diptera, Drosophilidae). J. Insect Behav., 9, 329-338.

NORRY, F. M., VILARDI, J. C., FANARA, J. J. AND HASSON, E. 1995a. Courtship success and multivariate analysis of sexual selection on morphometric traits in Drosophila buzzatii (Diptera: Drosophilidae). J. Insect Behav., 8, 219-229.

NORRY, F. M., VILARD1, J. C., FANARA, J. J., HASSON, E. AND RODRIGUEZ, C. 1995b. An adaptive chromosomal polymorphism affecting size-related traits, and longevity selection in a natural population of Drosophila buzzatii. Genetica, 96, 285-291.

NORRY, F. M., VILARDI, J. C. AND HASSON, E. 1997. Genetic and phenotypic correlations among site-related traits, and heritability variation between body parts in Drosophila buzzatii. Genetica, in press.

PARTRIDGE, L. AND FOWLER, K. 1993. Responses and correlated responses to artificial selection on thorax length in Drosophila melanogaster. Evolution, 47, 213-226.

PREVOSTI, A. 1967. Inversion heterozygosity and selection for wing length in Drosophila subobscura. Genet. Res., 10, 81-93.

PROUT, T. AND BARKER,J. S. F. 1989. Ecological aspects of the heritability of body size in Drosophila buzzatii. Genetics, 123, 803-813.
RICE, w. R. 1989. Analyzing tables of statistical tests. Evolution, 43, 223-225.

ROFF, D. A. 1995. The estimation of genetic correlations from phenotypic correlations: a test of Cheverud's conjecture. Heredity, 74, 481-490.

ROFF, D. A. 1996. The evolution of genetic correlations: an analysis of patterns. Evolution, 50, 1392-1403.

ROHLF, F. J. 1988. NTSYS-pc: Numerical Taxonomy and Multivariate Analysis System (Version 1.40). Exeter Publishing Ltd, New York.

RUIZ, A., SANTOS, M., BARBADILlA, A., QUEZADA-DIAZ, J. E., HASSON, E. AND FONTDEVILA, A. 1991. Genetic variance for body size in a natural population of Drosophila buzzatii. Genetics, 128, 739-750.

SANTOS, M., RUIZ, A., BARBADILla, A., QUEZADA-DlAZ, J. E., HASSON, E. AND FONTDEVILA, A. 1988. The evolutionary history of Drosophila buzzatii. XIV. Larger flies mate more often in nature. Heredity, 61, 255-262.

SANTOS, M., RUIZ, A., QUEZADA-DIAZ, J. E., BARBADILlA, A. AND FONTDEVILA, A. 1992. The evolutionary history of Drosophila buzzatii. XX. Positive phenotypic covariance between field adult fitness components and body size. $J$. Evol. Biol., 5, 403-422.

SCHLUTER, D. AND SMITH, J. N. M. 1986. Genetic and phenotypic correlations in a natural population of song sparrows. Biol. J. Linn. Soc., 29, 23-36.

SIMONS, A. M. AND ROFF, D. A. 1996. The effect of a variable environment on the genetic correlation structure in a field cricket. Evolution, 50, 267-275.

STALKER, H. D. 1980. Chromosome studies in wild populations of Drosophila melanogaster. II. Relationships of inversion frequencies to latitude, season, wing-loading and flight activity. Genetics, 95, 211-223.

THOMAS, R. H. AND BARKER, J. S. F. 1993. Quantitative genetic analysis of the body size and shape of Drosophila buzzatii. Theor. Appl. Genet., 85, 598-608.

TURELli, M. 1988. Phenotypic evolution, constant covariances, and the maintenance of additive variance. Evolution, 42, 1342-1347. 